\title{
Predicting the phases of a two-dimensional hard-rod system with real-space self-consistent field theory
}

\author{
Russell B. Thompson \\ Department of Physics, University of Waterloo, Waterloo, Ontario, Canada N2L $3 G 1$
}

(Received 19 July 2006; published 16 October 2006)

\begin{abstract}
Polymer self-consistent field theory numerical tools are applied to a two-dimensional hard-rod colloidal system. Rods are represented through an interaction site model density functional theory that is derived and expressed from a self-consistent field theory perspective. A weighted density approximation is used within the density functional theory, and the phase space is sampled without bias for any particular morphology. A completely ordered crystal phase is found as well as a liquid crystal state.
\end{abstract}

DOI: 10.1103/PhysRevE.74.041501

PACS number(s): 82.70.Dd, 61.30.Cz, 89.75.Fb

\section{INTRODUCTION}

Predicting the structures of materials based on the properties of the material constituents is of obvious importance in condensed matter physics and materials science and engineering. Progress in this direction has been made in the soft matter area of polymer physics using a suite of computational tools developed within numerical self-consistent field theory (SCFT) [1-8]. These computational advances are well summarized in the monograph of Fredrickson [9]. Reviews of SCFT and its applications can be found in Refs. [2,10-12].

A method of transporting these polymer structure predictive techniques to other materials, such as colloids, has recently been proposed [13]. This method uses SCFT advances on an interaction site model classical density functional theory (DFT) $[14,15]$, although it is quite generally valid in principle for almost any DFT. In [13], a simplest case, anisotropic colloid, was considered, comprised of two disks fused together to form a single $\mathrm{N}_{2}$-like colloid; this system was then solved in two dimensions (2D). A fully ordered crystal phase was found, as well as a plastic crystal phase, in addition to the homogeneous gas and liquid states. A phase diagram was presented that delineated regions of each, as well as the nature of the transitions within the mean-field model.

The purpose of the present paper is to give a full mathematical description of the general interaction-site SCFTDFT hybrid model discussed in a previous paper [13], as well as a more in-depth discussion of the numerical method. The model is applied here to another 2D system, this time a hard-rod colloid comprised of four interaction-site disks. Also, a different technique for exploring the phase behavior is employed, where an accurate phase diagram is not presented, but rather, larger cell calculations are performed that permit the direct observation of macrophase separation in the system. This is another technique borrowed from polymer SCFT [5].

A qualitative difference in results is found between the $\mathrm{N}_{2}$-like system and the hard-rod system purely due to the change in colloid geometry. A fully ordered crystal phase is still found in the rod system, but a liquid crystal phase now appears, while the plastic crystal phase is absent.

\section{THEORY}

A system is considered that is composed of $n$ molecules formed by $N$ spherical interaction sites (monomers) each, in a rod shape as shown in Fig. 1. The one monomer number density operator can be written as [16]

$$
\hat{\varrho}(\mathbf{r})=\sum_{i=1}^{n} \sum_{j=1}^{N} \delta\left(\mathbf{r}-\mathbf{r}_{i j}\right)
$$

As shown in Fig. 1, the separation between monomers is fixed at a distance $l$, so

$$
\mathbf{r}_{i j}+l \mathbf{u}_{i}=\mathbf{r}_{i(j+1)},
$$

where $\mathbf{u}_{i}$ denotes the unit vector pointing in the direction of the $i$ th molecule. Bearing in mind the constraint (2), the partition function for this rigid-rod system in the canonical ensemble will be

$$
\begin{aligned}
Z= & \frac{1}{\Omega^{n} \Lambda^{D n} n !} \int \prod_{i=1}^{n} d \mathbf{u}_{i} \prod_{j=1}^{N} d \mathbf{r}_{i j} \frac{\delta\left(\mathbf{r}_{i j}-\mathbf{r}_{i(j+1)}+l \mathbf{u}_{i}\right)}{\delta\left(\mathbf{r}_{i N}-\mathbf{r}_{i(N+1)}+l \mathbf{u}_{i}\right)} \\
& \times \exp \left\{-\mathcal{V}_{\text {mono }}^{*}[\hat{\varrho}]\right\},
\end{aligned}
$$

where $D$ is the dimensionality of the system, $\Lambda$ is the de Broglie wavelength, and $\Omega \equiv \int d \mathbf{u}$ is the integral over $d \mathbf{u}$, being shorthand for integration over angular degrees of freedom. In two dimensions, $\Omega=2 \pi$, and in three dimensions, $\Omega=4 \pi$. The constraint (2) appears as the numerator $\delta$ function, whereas the denominator $\delta$ function is just to remove the extra bond from the product over $j$ (there should be one less bond than interaction site). $\mathcal{V}_{\text {mono }}^{*}[\hat{\varrho}]$ is the monomermonomer interaction in terms of the density operator (1). Any particle-based Hamiltonian can be written in terms Eq.

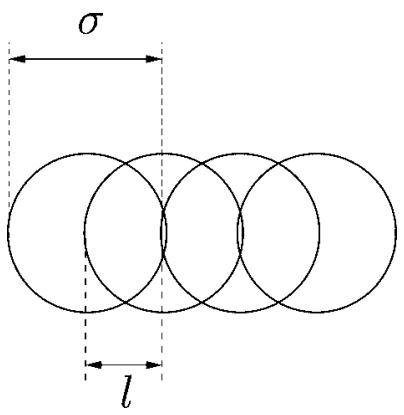

FIG. 1. A rod molecule composed of $N=4$ spheres with centers separated by a distance $l=0.5 \sigma$. 
(1) $[2,16]$, but excluded-volume interactions involving infinities do not lend themselves to expression in a field-based representation. Therefore, $\mathcal{V}_{\text {mono }}^{*}[\hat{\varrho}]$ will not be specified at this stage. Rather, a density functional approximation will be used later. The asterix on $\mathcal{V}_{\text {mono }}^{*}$ is a reminder that this potential is the interaction between two monomers that are contained within a rod, rather than the interaction between bare monomers.

Noting the identity [17]

$$
F[\hat{\varrho}]=\int \mathcal{D} \varrho \delta(\varrho(\mathbf{r})-\hat{\varrho}(\mathbf{r})) F[\varrho(\mathbf{r})]
$$

allows Eq. (3) to be written as

$$
\begin{aligned}
Z= & \frac{1}{\Omega^{n} \Lambda^{D n} n !} \int \prod_{i=1}^{n} d \mathbf{u}_{i} \prod_{j=1}^{N} d \mathbf{r}_{i j} \mathcal{D} \varrho \delta(\varrho-\hat{\varrho}) \\
& \times \frac{\delta\left(\mathbf{r}_{i j}-\mathbf{r}_{i(j+1)}+l \mathbf{u}_{i}\right)}{\delta\left(\mathbf{r}_{i N}-\mathbf{r}_{i(N+1)}+l \mathbf{u}_{i}\right)} \exp \left\{-\mathcal{V}_{\text {mono }}^{*}[\varrho]\right\} .
\end{aligned}
$$

Note the replacement of $\hat{\varrho}$ with $\varrho$ in $\mathcal{V}_{\text {mono }}^{*}$. Taking the identity [2]

$$
\delta(\varrho(\mathbf{r})-\hat{\varrho}(\mathbf{r}))=\int_{-i \infty}^{i \infty} \mathcal{D} W \exp \left\{\int d \mathbf{r} W(\mathbf{r})[\varrho(\mathbf{r})-\hat{\varrho}(\mathbf{r})]\right\}
$$

means that Eq. (5) can be written as

$$
\begin{aligned}
Z= & \frac{1}{\Omega^{n} \Lambda^{D n} n !} \int \prod_{i=1}^{n} d \mathbf{u}_{i} \prod_{j=1}^{N} d \mathbf{r}_{i j} \mathcal{D} \varrho \mathcal{D} W \delta(\varrho-\hat{\varrho}) \\
& \times \frac{\delta\left(\mathbf{r}_{i j}-\mathbf{r}_{i(j+1)}+l \mathbf{u}_{i}\right)}{\delta\left(\mathbf{r}_{i N}-\mathbf{r}_{i(N+1)}+l \mathbf{u}_{i}\right)} \exp \left[-\int d \mathbf{r} W(\mathbf{r}) \hat{\varrho}(\mathbf{r})\right] \\
& \times \exp \left\{-\mathcal{V}_{\text {mono }}^{*}[\varrho]+\int d \mathbf{r} W(\mathbf{r}) \varrho(\mathbf{r})\right\} .
\end{aligned}
$$

Considering the integral in the argument of the first exponential of Eq. (7) together with the definition of the density operator (1) gives

$$
\int d \mathbf{r} W(\mathbf{r}) \varrho(\mathbf{r})={ }_{i=1}^{n}{ }_{j=1}^{N} \int d \mathbf{r} W(\mathbf{r}) \delta\left(\mathbf{r}-\mathbf{r}_{i j}\right)={ }_{i=1}^{n}{ }_{j=1}^{N} W\left(\mathbf{r}_{i j}\right),
$$

so that

$$
\exp \left[-\int d \mathbf{r} W(\mathbf{r}) \hat{\varrho}(\mathbf{r})\right]=\prod_{i=1}^{n} \exp \left[-{ }_{j=1}^{N} W\left(\mathbf{r}_{i j}\right)\right] .
$$

A partition function of a single rod subject to the field $W(\mathbf{r})$ can be defined as

$$
\begin{aligned}
Q & \equiv \int d \mathbf{u} \prod_{j=1}^{N} d \mathbf{r}_{j} \frac{\delta\left(\mathbf{r}_{j}-\mathbf{r}_{(j+1)}+l \mathbf{u}\right)}{\delta\left(\mathbf{r}_{N}-\mathbf{r}_{(N+1)}+l \mathbf{u}\right)} \exp \left[-{ }_{k=1}^{N} W\left(\mathbf{r}_{k}\right)\right] \\
& =\int d \mathbf{u} d \mathbf{r} \exp \left[-{ }_{k=1}^{N}-W[\mathbf{r}+(k-1) l \mathbf{u}]\right] .
\end{aligned}
$$

Writing Eq. (7) in terms of Eqs. (8) and (9) gives

$$
\begin{aligned}
Z= & \frac{V^{n}}{\Lambda^{D n} n !} \int \mathcal{D} \varrho \mathcal{D} W\left(\frac{Q}{\Omega V}\right)^{n} \exp \left\{-\mathcal{V}_{\text {mono }}^{*}[\varrho]\right. \\
& \left.+\int d \mathbf{r} W(\mathbf{r}) \varrho(\mathbf{r})\right\}
\end{aligned}
$$

where $V$ is the volume of the system for $D=3$ or the area for $D=2$. The volume has been introduced here to simplify the notation later on. The partition function (10) is at this point an identity for the particle-based partition function (3). Making a mean-field (saddle function) approximation of Eq. (10) gives a partition function of

$$
Z \approx \frac{V^{n}}{\Lambda^{D n} n !}\left(\frac{Q}{\Omega V}\right)^{n} \exp \left\{-\mathcal{V}_{\text {mono }}^{*}[\rho]+\int d \mathbf{r} w(\mathbf{r}) \rho(\mathbf{r})\right\},
$$

where $\rho(\mathbf{r})$ and $w(\mathbf{r})$ are the functions for which the integrand of Eq. (10) attains its maximum. From the partition function (11), it is straightforward to write the free energy of the system as

$$
\begin{aligned}
\frac{F}{k_{B} T}= & -n \ln \left(\frac{V}{\Lambda^{D}}\right)+\ln n !-n \ln \left(\frac{Q}{\Omega V}\right) \\
& +\mathcal{V}_{\text {mono }}^{*}[\rho]-\int d \mathbf{r} w(\mathbf{r}) \rho(\mathbf{r}),
\end{aligned}
$$

where $T$ is the temperature and $k_{B}$ is Boltzmann's constant. Using Stirling's approximation, rephrasing, and dropping some constants and linear functions of $n$ gives a free energy functional of

$$
\begin{aligned}
\frac{F \sigma^{D}}{k_{B} T V}= & \frac{\sigma^{D}}{V}\left\{\frac{\rho_{s} V}{N}\left[\ln \left(\frac{\rho_{s} \sigma^{D}}{N}\right)-\ln \left(\frac{Q}{\Omega V}\right)\right]\right. \\
& \left.-\int d \mathbf{r} w(\mathbf{r}) \rho(\mathbf{r})+\frac{F_{\mathrm{ex}}[\rho]}{k_{B} T}\right\},
\end{aligned}
$$

where $\sigma$ is the diameter of a monomer sphere and $\rho_{s}$ is the overall number density of spheres in the system. Also, since $\mathcal{V}_{\text {mono }}^{*}[\rho]$ is playing the role of the contribution to the free energy of the excluded volume of the spheres, it has been relabeled as $F_{\text {ex }}[\rho] / k_{B} T$, indicating that it is the (dimensionless) excess free energy beyond the ideal gas. For the case of rods comprised of only one sphere, the fields $w(\mathbf{r})$ algebraically vanish and Eq. (13) becomes the usual density functional expression $F_{\text {id }}+F_{\text {ex }}$ that one would expect, where $F_{\text {id }}$ is the ideal gas free energy.

Just as with the hard-sphere case, a mean-field attractive term can be added to Eq. (13) to give

$$
\begin{aligned}
\frac{F \sigma^{D}}{k_{B} T V}= & \frac{\sigma^{D}}{V}\left\{\frac{\rho_{\mathrm{s}} V}{N}\left[\ln \left(\frac{\rho_{\mathrm{s}} \sigma^{D}}{N}\right)-\ln \left(\frac{Q}{\Omega V}\right)\right]-\int d \mathbf{r} w(\mathbf{r}) \rho(\mathbf{r})\right. \\
& \left.+\frac{1}{2} \int d \mathbf{r} d \mathbf{r}^{\prime} \rho(\mathbf{r}) \phi_{\mathrm{att}}\left(\mathbf{r}-\mathbf{r}^{\prime} \mid\right) \rho\left(\mathbf{r}^{\prime}\right)+\frac{F_{\mathrm{ex}}[\rho]}{k_{B} T}\right\},
\end{aligned}
$$

where $\phi_{\text {att }}(r)$ is a two-body attractive potential. Together with the free energy functional (14), we can rewrite Eq. (9) in the final form 


$$
Q=\int d \mathbf{u} d \mathbf{r} \prod_{k=0}^{N-1} f_{k}(\mathbf{r}, \mathbf{u}),
$$

where

$$
f_{k}(\mathbf{r}, \mathbf{u})=\mathrm{e}^{-w(\mathbf{r}+k l \mathbf{u})} .
$$

The free energy functional is varied with respect to $\rho(\mathbf{r})$ and $w(\mathbf{r})$ to find a self-consistent solution to Eq. (14). The resulting equation are

$$
\begin{gathered}
\rho(\mathbf{r})=\frac{\rho_{s} V}{N Q} \int d \mathbf{u} \prod_{p=0}^{N-1} \prod_{k=0}^{N-1} f_{(k-p)}(\mathbf{r}, \mathbf{u}), \\
w(\mathbf{r})=\int d \mathbf{r}^{\prime} \phi_{\mathrm{att}}\left(\left|\mathbf{r}-\mathbf{r}^{\prime}\right|\right) \rho\left(\mathbf{r}^{\prime}\right)+\frac{\delta F_{\mathrm{ex}}[\rho] / k_{B} T}{\delta \rho(\mathbf{r})} .
\end{gathered}
$$

For $F_{\text {ex }}$, one wants to select a spherically symmetric potential for a hard repulsion. As a simple approximation, the Tarazona hard-sphere-weighted density approximation (WDA) functional [18] is chosen here, but more accurate WDA functionals based on good knowledge of the uniform fluid twobody direct correlation function for the interaction sites could also be used $[19,20]$, as could completely different types of DFT's [21]. The Tarazona functional is

$$
\frac{F_{\mathrm{ex}}}{k_{B} T}=\int d \mathbf{r} \rho(\mathbf{r}) \Psi[\bar{\eta}(\mathbf{r})],
$$

where $\Psi$ is a free energy per particle that can be acquired from an appropriate equation of state. It is a function of $\bar{\eta}$, which is the local "smoothed" packing fraction, defined by

$$
\bar{\eta}(\mathbf{r})=\frac{\pi}{2 D} \sigma^{D} \bar{\rho}(\mathbf{r})
$$

for $D=2$ or $D=3$ dimensions, with the smoothed density $\bar{\rho}(\mathbf{r})$ given by

$$
\bar{\rho}(\mathbf{r})=\int d \mathbf{r}^{\prime} \rho\left(\mathbf{r}^{\prime}\right) \mathcal{W}\left(\left|\mathbf{r}-\mathbf{r}^{\prime}\right|\right) .
$$

$\mathcal{W}(r)$ is a weighting function which introduces nonlocality into the WDA density functional theory. It can be selected to be consistent with the uniform direct correlation function of the monomers, but in this paper the simple and less quantitative method of Tarazona will be used, in which $\mathcal{W}(r)$ is taken as the step function

$$
\mathcal{W}(r)= \begin{cases}\frac{3}{\pi \sigma^{(D+1)}}(\sigma-r), & r \leq \sigma, \\ 0, & r>\sigma,\end{cases}
$$

for $D=2$ or $D=3$ dimensions. The prefactor in Eq. (22) enforces the normalization condition

$$
\int d \mathbf{r} \mathcal{W}(r)=1
$$

Overall, then, the self-consistent equations will be

$$
\rho(\mathbf{r})=\frac{\rho_{s} V}{N Q} \int d \mathbf{u} \prod_{p=0}^{N-1} \prod_{k=0}^{N-1} f_{(k-p)}(\mathbf{r}, \mathbf{u}),
$$

$$
\begin{aligned}
w(\mathbf{r})= & \Psi[\bar{\eta}(\mathbf{r})]+\int d \mathbf{r}^{\prime} \eta\left(\mathbf{r}^{\prime}\right) \frac{d \Psi[\bar{\eta}]}{d \bar{\eta}} \mathcal{W}\left(\left|\mathbf{r}-\mathbf{r}^{\prime}\right|\right) \\
& +\int d \mathbf{r}^{\prime} \phi_{\text {att }}\left(\left|\mathbf{r}-\mathbf{r}^{\prime}\right|\right) \rho\left(\mathbf{r}^{\prime}\right)
\end{aligned}
$$

where $\eta$ is defined in terms of $\rho$ just as $\bar{\eta}$ was defined in terms of $\bar{\rho}$ in Eq. (20). If the monomers are overlapping (fused interaction sites), the packing fraction (20) should be modified accordingly [22]. The excess free energy (19) can also be modified by a prefactor so that it is consistent in the unified atom limit $l \rightarrow 0$. This causes a slight modification in the self-consistent equation (25) as discussed in [22]. It should be noted that the choice of the Tarazona functional also has the advantage that the present theory reduces to that of Oxtoby and co-workers when written in the grand canonical SCFT formalism $[23,24]$ while ignoring orientational dependences [25-30].

The free energy per particle $\Psi$ should be chosen as spherically symmetric, in keeping with the interaction-site model approach, according to the molecules involved. To lowest order, a generic equation of state that enforces a hard core should suffice. In three dimensions, the CarnahanStarling expression [31]

$$
\Psi(\eta)=\frac{4 \eta-3 \eta^{2}}{(1-\eta)^{2}}
$$

could be chosen, and in two dimensions, an expression for hard disks is [22]

$$
\Psi(\eta)=-\ln (1-\eta)+\frac{\eta}{1-\eta}
$$

The attractive potential $\phi_{\text {att }}(r)$ in Eq. (25) is chosen in this work to be the attractive part of a cutoff Lennard-Jones potential, given by

$$
\phi_{\text {att }}(r)= \begin{cases}U\left(r_{\min }\right)-U\left(r_{c}\right), & r \leq r_{\min }, \\ U(r)-U\left(r_{c}\right), & \text { otherwise, }\end{cases}
$$

where

$$
U(r)=\frac{4 \varepsilon}{k_{B} T}\left[\left(\frac{\sigma}{r}\right)^{12}-\left(\frac{\sigma}{r}\right)^{6}\right]
$$

and $r_{\min }=2^{1 / 6} \sigma$ with $r_{c}$ the cutoff value chosen as roughly half the system size.

\section{NUMERICAL METHOD}

The real-space method used to calculate the (meta)stable phases of the hard-rod system is based on the polymer SCFT techniques described by Drolet and Fredrickson $[1,2,9]$. The successful transfer of these techniques has been demonstrated in a previous publication by the present author [13]. All spatially varying functions are discretized on a regular 


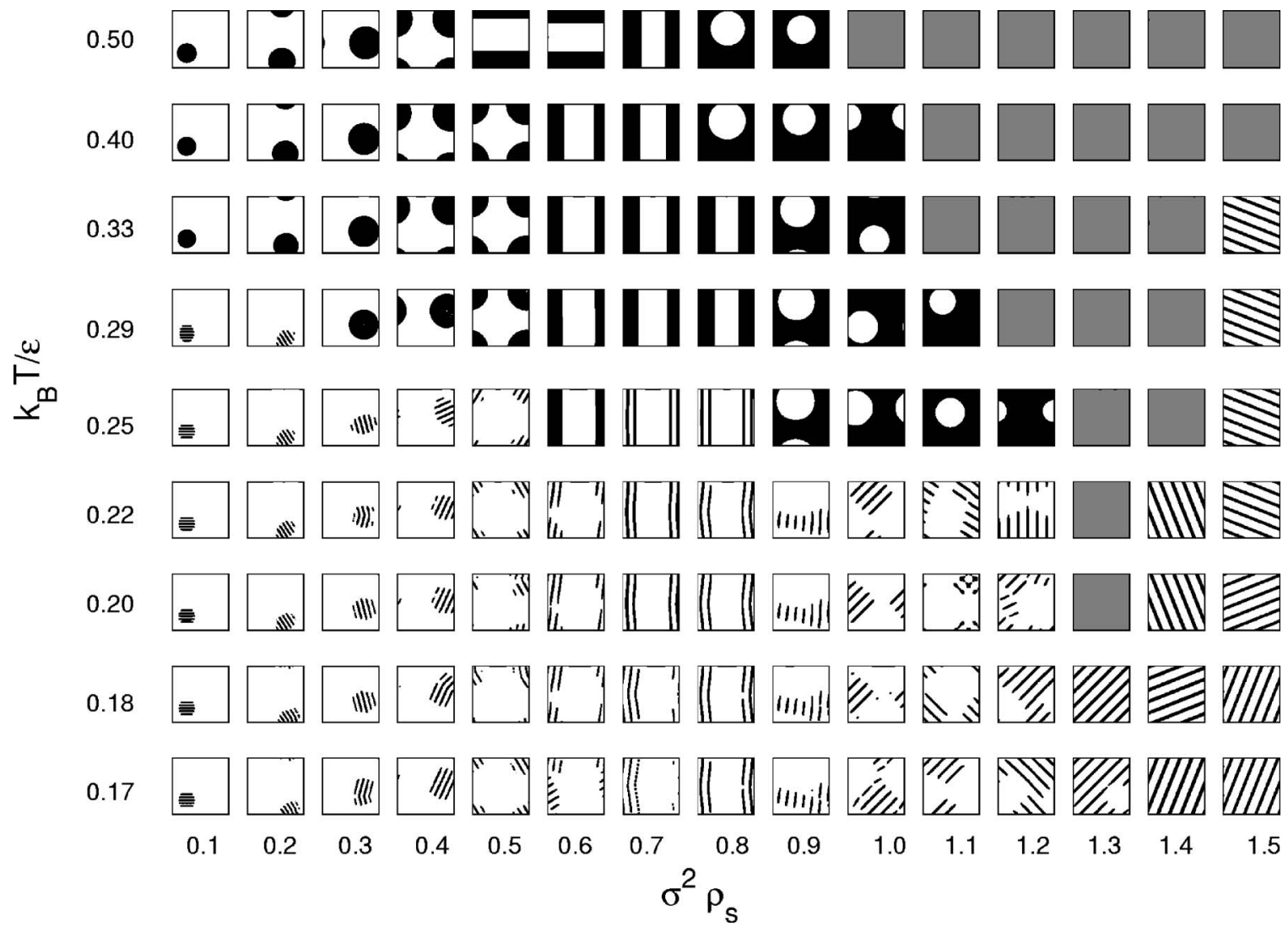

FIG. 2. Data results of runs showing a "sketch" of possible morphologies for the hard-rod system. Black indicates presence of "monomer" density and white the absence. Gray panels are completely uniform phases with no phase separation. The overall density $\rho_{S}$ increases on the $x$ axes, and $k_{B} T / \varepsilon$ increases on the $y$ axis.

grid; a constant value for the discretized field $w(\mathbf{r})$ subject to substantial random noise is taken at the outset. Using this field, the propagator (16) is assigned, which in turn allows the calculation of $Q$ and the density field $\rho(\mathbf{r})$ through Eqs. (15) and (24), respectively. The smoothed density and packing fraction, Eqs. (21) and (20), can then be computed, allowing one to determine the free energy per particle, given by either Eq. (26) or (27), depending on the dimensionality of the problem. Finally, Eq. (25) gives a new value for the chemical potential field $w(\mathbf{r})$ on which the whole process can be repeated. Iteration is continued until the total deviation between the input and output fields differs by less than some acceptable tolerance. In actuality, such a direct substitution algorithm is not stable, and the new and old fields are combined in a simple mixing scheme described in [7]. The total deviation is defined as a normalized scalar product of a deviation function as described in $[7,32]$; in cases where this definition behaves poorly, it is replaced by the definition of $\mathrm{Ng}$ [33]. In order to speed convergence, a modification of polymeric SCFT Anderson mixing is also used [7]. The modification consists of doing single Anderson steps at set intervals instead of the simple mixing step. This interdigitation of iteration methods is in contrast to the method described in [7], where simple mixing is continuously used until a certain accuracy is reached and thenAnderson mixing is used exclusively. Although the latter is effective for poly- mers, the modified interdigitation approach mentioned here is much better for the present colloids.

The idea of using Fourier transforms to expedite realspace SCFT algorithms was suggested by Rasmussen and co-workers in the context of polymeric systems $[3,4]$. In that context, the modified diffusion equation was solved using this pseudospectral approach. There is clearly no diffusion partial differential equation here, but instead there are convolution integral equations, such as Eqs. (21) and (25), for which Fourier methods greatly speed calculations. Similarly, Fourier interpolation methods, as described by Ceniceros and Fredrickson [6], can be used to efficiently increase the grid density to check the accuracy of results without doing independent, higher-resolution, runs.

After convergence is reached, the stable $w(\mathbf{r})$ and $\rho(\mathbf{r})$ fields are substituted into the free energy expression (14). This process can be repeated for many different initial random $w(\mathbf{r})$ configurations with the lowest free energy result being taken as the equilibrium morphology. In the previous work [13], the whole iterative procedure was then nested in a simplex minimization routine that varies the calculational box size and aspect ratio to find the lowest possible free energy based on cell commensurability. In this work, the box size is held fixed as a square with periodic boundary conditions and chosen to be sufficiently large such that incommensurability effects are not important. For a given overall density $\sigma^{2} \rho_{s}$, the length of a side of a box is taken to be 


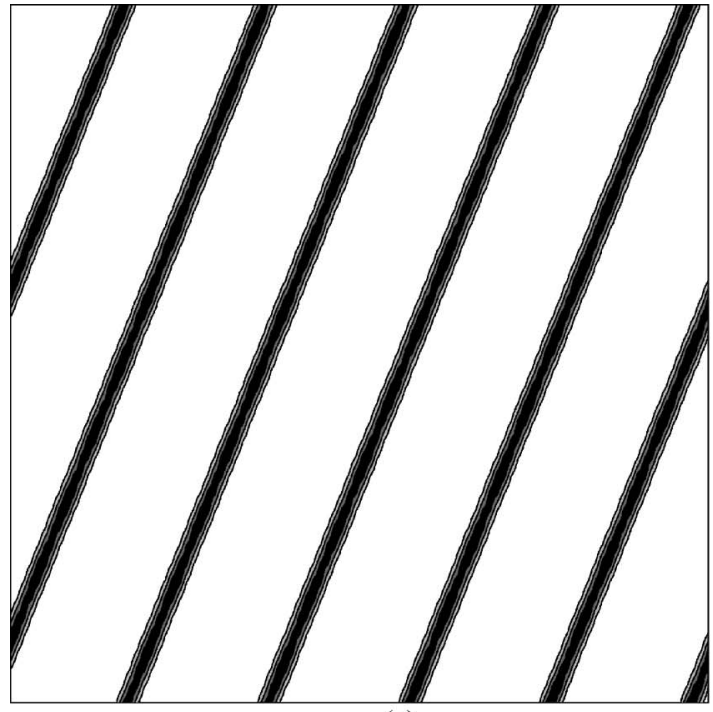

(a)

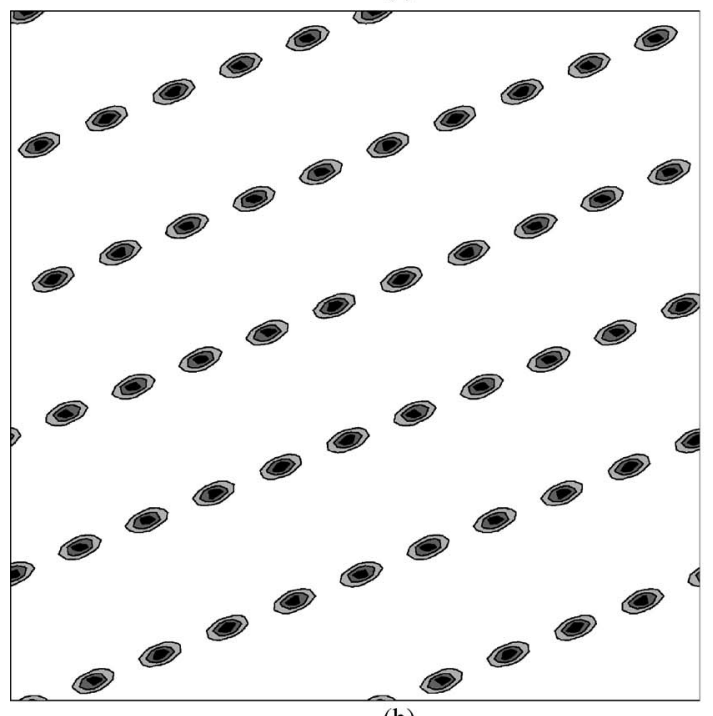

(b)
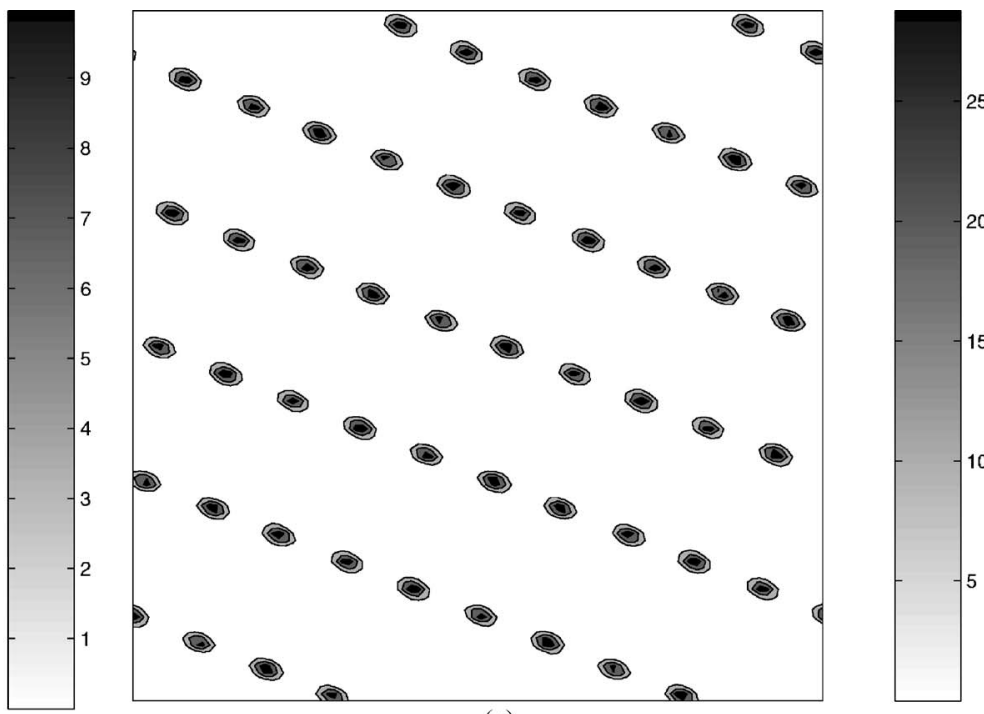

(c)
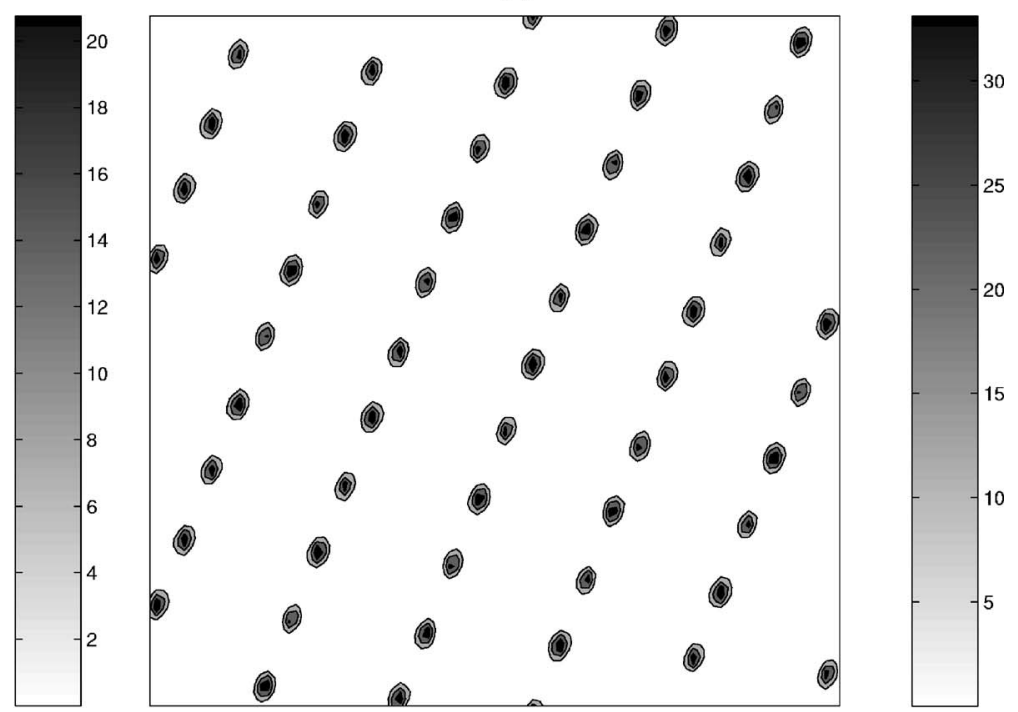

(d)

FIG. 3. Crystallization of a 2D hard-rod system at density $\sigma^{2} \rho_{s}=1.5$ and temperatures (a) $k_{B} T / \varepsilon=$ (a) 0.17 , (b) 0.15 , (c) 0.13 , and (d) 0.11 . The color bars indicate the local monomer densities, $\sigma^{2} \rho(\mathbf{r})$.

$$
\frac{L}{\sigma}=\sqrt{\frac{40}{\sigma^{2} \rho_{s}}} .
$$

The number 40 appears in the numerator of Eq. (30) since 40 disk monomers were taken in each calculation. The use of a larger box size instead of varying the aspect ratio of a smaller box as in [13] means that the free energy per volume is calculated at a lower accuracy. This is not a problem here since a phase diagram will not be constructed. Rather, the morphologies at various points of parameter space will be individually examined, as explained in the Results and Discussion section.

\section{RESULTS AND DISCUSSION}

It has been shown previously that the theory outlined above can be used to construct phase diagrams [13]. This is based on comparing the free energies of different phases to determine the stable morphology and using double-tangent constructions to dilineate regions of two-phase coexistence. Alternatively, the SCFT-DFT approach could equally well be implemented using a grand canonical description $[23,24]$ and coexisting regions would be found by comparing chemical potentials. The canonical and grand canonical approaches must, of course, yield the same results. The two-phase regions are particularly important as they are the hallmark of first-order phase transitions [34]. Alternatively, to determine phases, coexistence regions, and therefore the nature of transitions, a less computationally demanding but more approximate approach can be taken. In the canonical ensemble, for large enough calculational cells (boxes), macrophase separation can be directly observed through entirely local calculations [5], albeit as mentioned, in an approximate way [35]. This means that through a single choice of parameters and a single corresponding calculation, the morphology of the system may be determined together with knowledge about whether this point of the parameter space is in a two-phase 


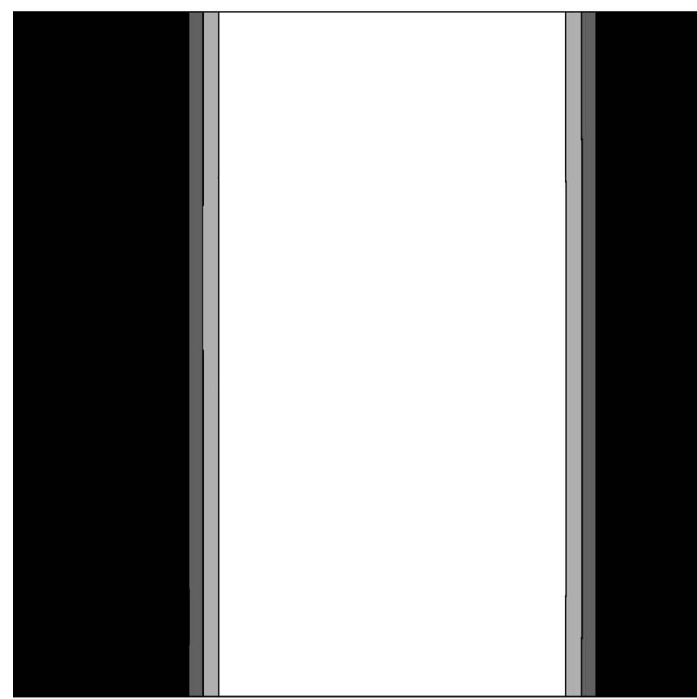

(a)

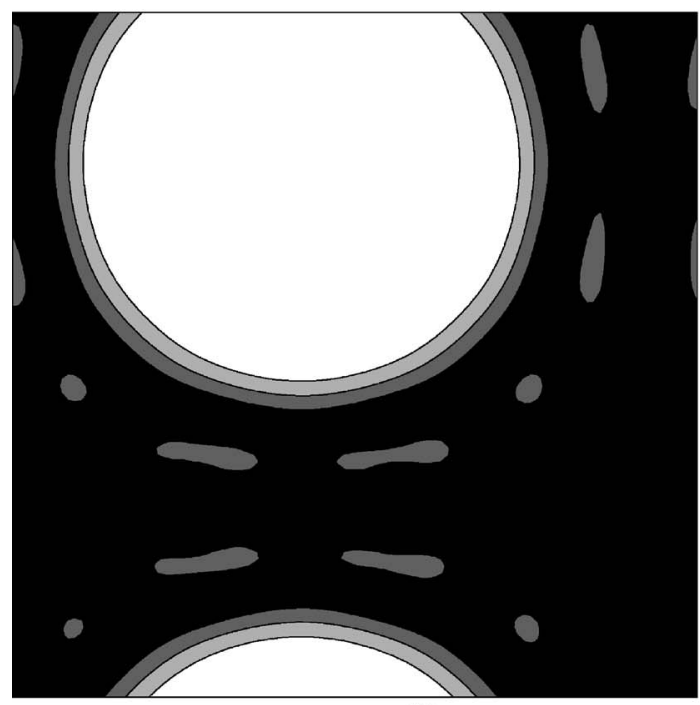

(b)
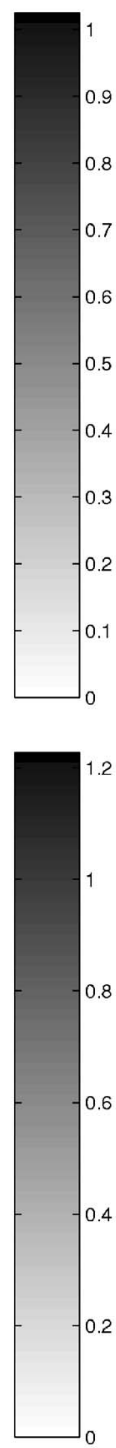

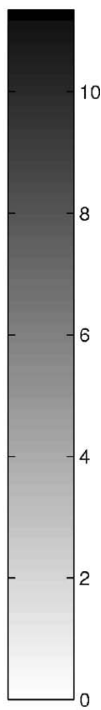

(c)

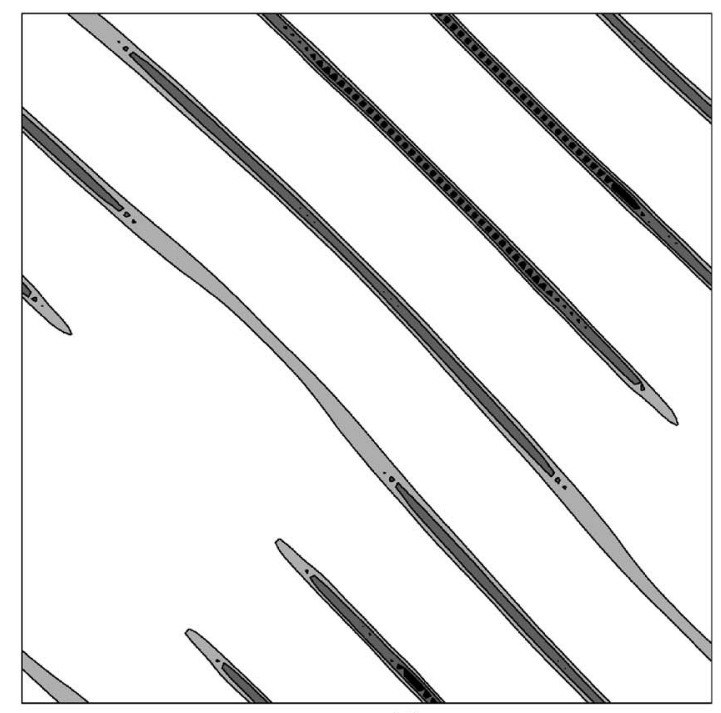

(d)

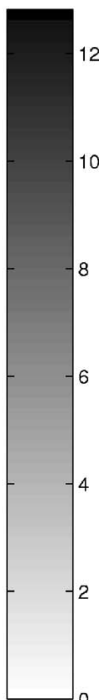

FIG. 4. Macrophase separation of a 2D hard-rod system for (a) $k_{B} T / \varepsilon=0.40$ and $\sigma^{2} \rho_{s}=0.6$, (b) $k_{B} T / \varepsilon=0.29$ and $\sigma^{2} \rho_{s}=0.9$, (c) $k_{B} T / \varepsilon$ $=0.18$ and $\sigma^{2} \rho_{s}=0.4$, and (d) $k_{B} T / \varepsilon=0.17$ and $\sigma^{2} \rho_{s}=1.2$. The color bars indicate the local monomer densities, $\sigma^{2} \rho(\mathbf{r})$.

region, and thus one obtains evidence about the nature of possible transitions [34]. This approach may be compared to the more rigorous method of comparing chemical potentials or constructing double tangents which are necessarily nonlocal in terms of the parameter space.

The particular system studied in this work is a twodimensional rod composed of $N=4$ spheres with centercenter separations of $l=0.5 \sigma-$ see Fig. 1 . The local approach is taken here for this system; instead of using free energies to construct a phase diagram as in [13], a number of sample calculations are performed and shown in Fig. 2. For each overall density $\sigma^{2} \rho_{s}$ and temperature $k_{B} T / \varepsilon$ in Fig. 2, a calculation from random fields was performed [36]. Three phases are observed in Fig. 2: two homogeneous phasescondensed and low density-and a lamellar type phase. This latter phase, being localized (crystallized) in one direction but homogeneous (fluid) in the other, corresponds to a liquid crystal (LC) phase with the rods being oriented parallel to the layers of the structure. An LC phase was not observed in the previous work by this author [13]; there an ordered phase and a plastic crystal phase were found in addition to the homogeneous states. The more pronounced anisotropy the colloids in the present case makes the appearance of an LC phase not unexpected, although it may also be due to the coarse equation of state. Should the anisotropy be further enhanced, a nematic phase could likely be observed. Bates and Frenkel [37] used Monte Carlo simulations to study a very similar system of $2 \mathrm{D}$ hard-rod spherocylinders (discorectangles). They found a nematic phase only for length to diameter aspect ratios $L / D \gtrsim 7$. The present system would correspond to $L / D=1.5$, and so the absence of a nematic phase is consistent with the results of Bates and Frenkel. Those authors did not however find an LC phase. It may be that the present LC phase is just a very weak crystal phase, 
just as the plastic crystal phase in [13] never actually lost all orientational order, thus being a very weak crystal phase.

True crystallization is found as the temperature is reduced further, as shown in Fig. 3. The four panels of Fig. 3 demonstrate the increasing localization of the "monomer" disks comprising the rods as temperature is lowered.

Returning to Fig. 2, many intermediate, macrophaseseparated phases are seen. Figure 4 shows blowups of some examples. Panels (a) and (b) show macrophase separation between homogeneous states (gas and liquid coexistence therefore) and panels (c) and (d) show separation in the LC phase. (The reader may note the resemblence between the present phase-separated structures and those of a simulated Lennard-Jones fluid [38].) The direct observation of phase separation means that, in principle, one may compute a single point of interest within a parameter space in order to discuss the morphology there without having to nonlocally check for phase separation [34,35]. This is particularly important for systems where each computational point of the phase diagram may take a (relatively) long time, such as in some three-dimensional calculations, or for colloids of a complex shape or severe anisotropy. Also, in SCFT-DFT calculations in real space, the morphology becomes reliably apparent quickly even for low-resolution calculations, but to compute free energies with sufficient accuracy for the construction of phase diagrams, much greater resolution, and therefore computational time, is needed. The approximate method of sketching the phase behavior shown here does not require knowledge of the free energies. If the model is qualitative in any case, even accurate computations of the free energies and phase diagrams result in only qualitative understanding of the phase behavior and is therefore not necessar- ily superior to a rough sketch. In any event, for systems with many components, double-tangent constructions are no longer practicle for determining multiphase coexistence and a grand canonical approach must be taken to compute an accurate phase diagram. The present, local approach, in the canonical ensemble is still effective for sketching the behavior of a system in these more complex circumstances.

\section{SUMMARY}

A hard-rod system in two dimensions comprised of four disks has been studied using an interaction-site model density functional theory and self-consistent field theory methods. Liquid crystal and fully crystal phases are found in addition to the usual gas and liquid homogeneous phases. A survey of the phase behavior has been made that shows regions of two-phase coexistence through direct observation of a larger cell size in the canonical ensemble. This approach, which does not require accurate free energy calculations, could be particularly useful for more computationally demanding systems. Other systems that could be studied include fully three-dimensional systems and colloids with greater orientational degrees of freedom or internal degrees of freedom.

\section{ACKNOWLEDGMENTS}

This work was supported by the National Sciences and Engineering Research Council (NSERC) of Canada. A major part of the SCFT-DFT calculations was performed using SHARCNET.
[1] F. Drolet and G. H. Fredrickson, Phys. Rev. Lett. 83, 4317 (1999).

[2] G. H. Fredrickson, V. Ganesan, and F. Drolet, Macromolecules 35, 16 (2002).

[3] G. Tzeremes, K. Ø. Rasmussen, T. Lookman, and A. Saxena, Phys. Rev. E 65, 041806 (2002).

[4] K. Ø. Rasmussen and G. Kalosakas, J. Polym. Sci., Part B: Polym. Phys. 40, 1777 (2002).

[5] S. W. Sides and G. H. Fredrickson, Polymer 44, 5859 (2003).

[6] H. D. Ceniceros and G. H. Fredrickson, Multiscale Model. Simul. 2, 452 (2004).

[7] R. B. Thompson, K. Ø. Rasmussen, and T. Lookman, J. Chem. Phys. 120, 31 (2004).

[8] J. L. Barrat, G. H. Fredrickson, and S. W. Sides, J. Phys. Chem. B 109, 6694 (2005).

[9] G. H. Fredrickson, The Equilibrium Theory of Inhomogeneous Polymers, (Oxford University Press, Oxford, 2006).

[10] M. W. Matsen, in Soft Matter, edited by G. Gompper and M. Schick (Wiley-VCH, Weinheim, 2005).

[11] M. W. Matsen, J. Phys.: Condens. Matter 14, R21 (2002).

[12] F. Schmid, J. Phys.: Condens. Matter 10, 8105 (1998).

[13] R. B. Thompson, Phys. Rev. E 73, 020502(R) (2006).

[14] D. Chandler, J. D. McCoy, and S. J. Singer, J. Chem. Phys. 85, 5971 (1986).
[15] J. D. McCoy, S. J. Singer, and D. Chandler, J. Chem. Phys. 87, 4853 (1987).

[16] N. W. Ashcroft, in Density Functional Theory, edited by E. K. U. Gross and R. M. Dreizler (Plenum Press, New York, 1995).

[17] M. W. Matsen and M. Schick, Phys. Rev. Lett. 72, 2660 (1994).

[18] P. Tarazona, Mol. Phys. 52, 81 (1984).

[19] W. A. Curtin and N. W. Ashcroft, Phys. Rev. A 32, 2909 (1985).

[20] A. R. Denton and N. W. Ashcroft, Phys. Rev. A 39, 4701 (1989).

[21] R. Evans, in Fundamentals of Inhomogeneous Fluids, edited by D. Henderson (Marcel Dekker, New York, 1992).

[22] T. A. Cherepanova and A. V. Stekolnikov, Chem. Phys. 154, 41 (1991). There would seem to be an extra factor of $(L / \sigma)$ on the last term of the right-hand side of Eq. (19) of this reference.

[23] M. W. Matsen, Phys. Rev. Lett. 74, 4225 (1995).

[24] M. W. Matsen, Macromolecules 28, 5765 (1995).

[25] V. Talanquer and D. W. Oxtoby, J. Chem. Phys. 103, 3686 (1995)

[26] V. Talanquer and D. W. Oxtoby, Physica A 220, 74 (1995).

[27] V. Talanquer and D. W. Oxtoby, J. Chem. Phys. 113, 7013 (2000). 
[28] P. S. Christopher and D. W. Oxtoby, J. Chem. Phys. 117, 9502 (2002).

[29] P. S. Christopher and D. W. Oxtoby, J. Chem. Phys. 119, 10330 (2003).

[30] P. S. Christopher and D. W. Oxtoby, J. Chem. Phys. 121, 5005 (2004).

[31] N. F. Carnahan and K. E. Starling, J. Chem. Phys. 51, 635 (1969).

[32] F. Schmid and M. Müller, Macromolecules 28, 8639 (1995).

[33] K.-C. Ng, J. Chem. Phys. 61, 2680 (1974).

[34] M. R. Fisch and C. Rosenblatt, J. Phys. II 4, 103 (1994).

[35] Observing direct macrophase separation in an appropriately done, real-space, SCFT calculation is good evidence that the system does phase separate at that point of the parameter space. However, the reverse is not true, in that real-space SCFT results not showing phase separation are not a guarantee that separation does not take place at that point.

[36] Three runs from different random starting fields were done for each point, to be sure of the final result. As there was never any difference among the three runs at any point, only data from one set are presented. These data are all from the same random starting point.

[37] M. A. Bates and D. Frenkel, J. Chem. Phys. 112, 10034 (2000).

[38] L. G. MacDowell, V. K. Shen, and J. R. Errington, J. Chem. Phys. 125, 034705 (2006). 